\title{
On the Relationship of Geomagnetic Storms to the Density of the Upper Atmosphere
}

\author{
Kenneth MoE \\ Institute of Geophysics and Planetary Physics \\ University of California, Los Angeles
}

\begin{abstract}
The increase in the temperature and density of the upper atmosphere during geomagnetic storms is discussed. The latitude dependences of various phenomena related to storms are examined in an effort to determine the latitude dependence of the neutral heating, which is not well determined by the drag of satellites. The energy balance in the auroral zone during storms is also discussed.
\end{abstract}

\section{Introduction}

The variation of geomagnetic activity with latitude has always interested workers in geomagnetism, but it has also recently become of interest to atmospheric physicists because of the correlation of satellite drag with geomagnetic activity (Jacchia, 1961). This latitude dependence may also affect the helium balance of the atmosphere (Cole, $1962 \mathrm{~b}$ ). Two of the mechanisms which have been proposed to account for atmospheric heating during geomagnetic storms are the attenuation of magnetohydrodynamic waves in the ionosphere (Dessler, 1959), and Joule heating by the disturbance currents in the ionosphere (Cole, 1962 a). When the ionosphere is heated, the scale height increases, and some of the air at altitudes of 100 to $150 \mathrm{~km}$ (approximately) rises to higher altitudes, increasing the density in the regions where artificial satellites travel. Since micropulsations and magnetic storms both are latitude dependent, Cole (1962 a) predicted that the corresponding increase in atmospheric density would also be. Jacchia and Slowey (1963 b) confirmed Cole's prediction, although there was some evidence available earlier which suggested that this would be the case.

The most striking piece of earlier evidence is shown in Figure 1 (Meadows and Townsend, 1960). The three heavy lines represent densities determined from pressure gauges on rockets fired from Ft. Churchill (geomagnetic latitude, $68^{\circ}$ ), during the period 1956 to 1958. NN 3.17 (circles) was fired at night when no aurora was visible, while NN $3.18 \mathrm{~F}$ (squares) was fired into a visible aurora. Notice in NN $3.18 \mathrm{~F}$ the sudden increase in density at $120 \mathrm{~km}$., which is about $10 \mathrm{~km}$ above the average height of the lower boundaries of auroras. Also observe that the density at $200 \mathrm{~km}$ is four times as large during the auroral display as on the quiet night. These effects could not have been produced by solar ultraviolet heating during the sunlit hours, because the $10 \mathrm{~cm}$ solar flux was lower on the day of the aurora than on the quiet day (175 compared with 216). The heating by auroras is 


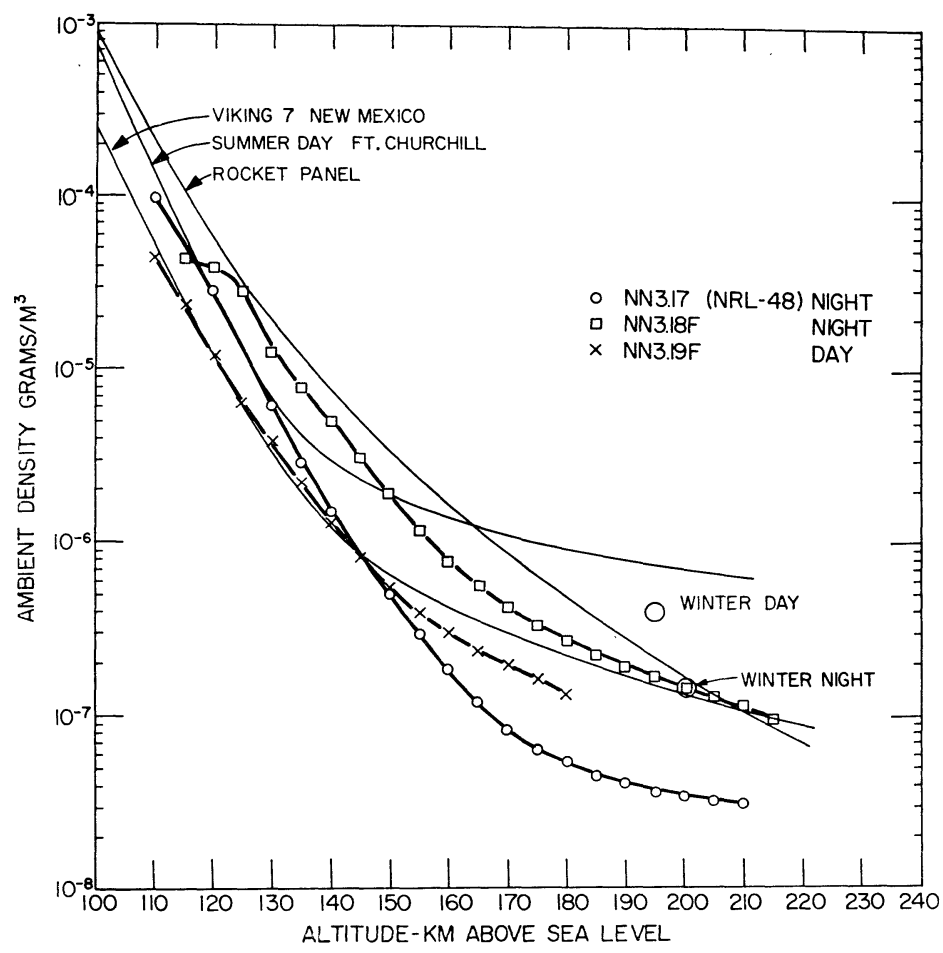

Fig. 1. Atmospheric density at Ft. Churchill, 1956-58 (after Meadows and Townsend).

localized, but during a magnetic storm, when intense and widespread auroras occur, a large increase in the average density over the whole auroral zone would be expected, because this local increase in density of a factor of four at $200 \mathrm{~km}$ occurred when $A_{p}$ was only 31.

\section{Satellite Observations of the Effect of Geomagnetic Storms on the Density and Temperature of the Upper Atmosphere}

During geomagnetically quiet times, the drag of satellites in orbits of high inclination has revealed that the density of the upper atmosphere does not vary with latitude by more than 30 percent: The variation with latitude found in the various studies of the orbital decay of Discoverer satellites (whose perigee heights were below $300 \mathrm{~km}$ ) ranged from zero to 30 percent (Groves, 1961, Smelt, 1961, Paetzold, 1963, May, 1964). For the Injun III satellite (at $250 \mathrm{~km}$ ) no latitude dependence of the density or temperature was discernible at quiet times (Jacchia and Slowey, 1963 b). At times of moderate geomagnetic activity (approximately $A_{p}<30$ ), the temperature and density above $200 \mathrm{~km}$ increase in proportion to the increase in $K_{p}$ (Jacchia and Slowey 1964; Newton et. al., 1964). During geomagnetic storms (approximately $A_{p}>30$ ), the temperature increases in proportion to $A_{p}$ (Jacchia, 1961), and the increase in temperature is strongly latitude-dependent (Jacchia and Slowey, $1962 \mathrm{~b}$ ).

Unfortunately, satellite drag data do not give good resolution in latitude, as is obvious from Figure 2. The Injun III satellite, which Jacchia and Slowey employed for studying the latitude-dependence of atmospheric heating during geomagnetic storms, had an eccen- 

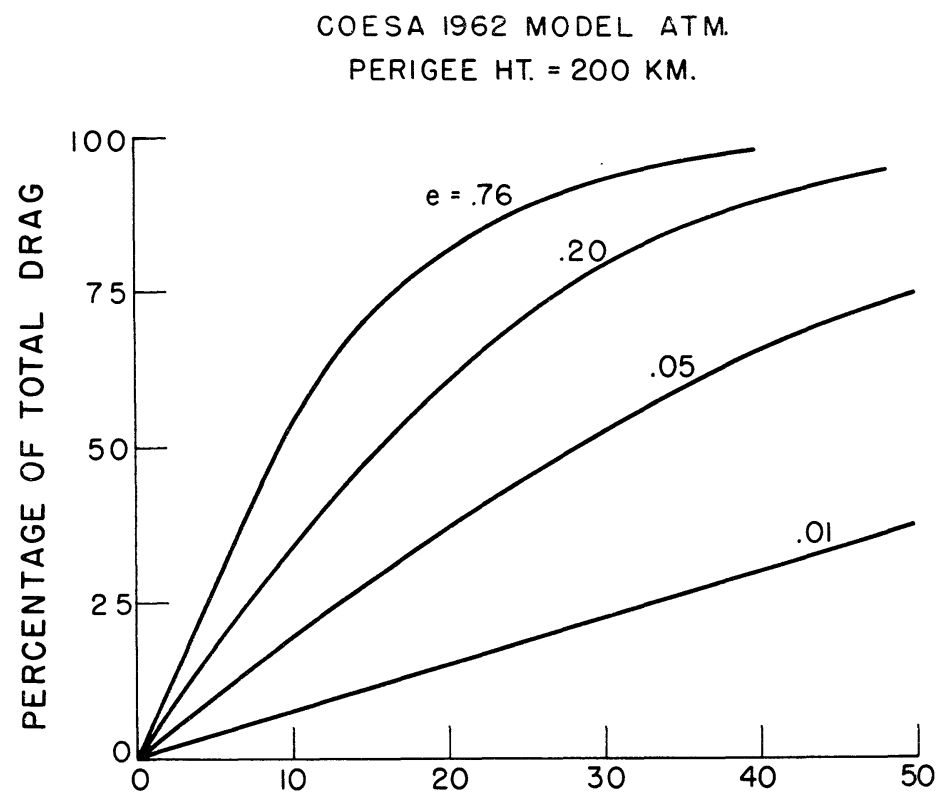

ANGULAR RANGE AROUND PERIGEE (DEG)

Fig. 2. Percentage of total air drag versus angular range around perigee for several eccentricities.

tricity of 0.16 , an inclination of $70.4^{\circ}$, and a perigee height of $250 \mathrm{~km}$. Therefore, ninety percent of its air drag occurred in about $50^{\circ}$ of true anomaly, centered at perigee. The corresponding range in geographic latitude, when perigee was near $70^{\circ}$ was about $12^{\circ}$. The spreading of the data in geomagnetic latitude was greater, because the geomagnetic dipole axis is offset $11.5^{\circ}$ from the geographic axis, and the drag data were derived from orbital data covering a considerable part of a day, considering that the second derivative of the observed positions had to be taken. This caused a further spreading of $23^{\circ}$. The Injun III drag data were therefore averaged over about $35^{\circ}$ of geomagnetic latitude, from $48^{\circ}$ to $83^{\circ}$.

\section{The Latitude Dependence of Geomagnetic Storms}

The poor resolution of satellite drag data have led the writer to examine the latitude dependences of other phenomena which occur during geomagnetic storms, in the hope that they would give a clue to the latitude dependence of the density. The first phenomenon examined was the latitude dependence of geomagnetic storms. Figure 3 shows the largest component of the magnetic storm ranges for the years near sunspot maximum, 1958 to 1960, plotted against geomagnetic latitude. Each data point represents the average range of storms observed at a single station for a period varying from three months to one year. Different time intervals were sometimes covered for different stations, so each storm range was normalized by dividing by the largest $A_{p}$ during that storm, before the range was averaged with the others. After the data had been graphed, a second normalization was performed to make the average ordinate from $0^{\circ}$ to $50^{\circ}$ geomagnetic latitude (correspond- 


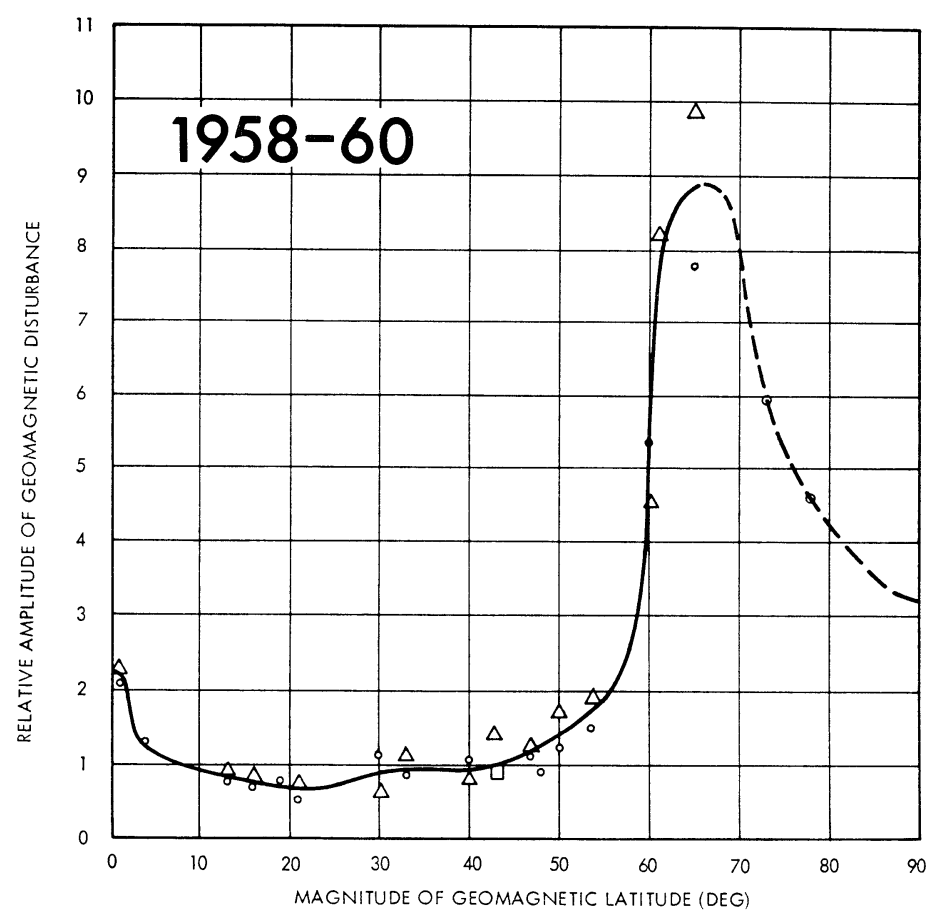

Fig. 3. Latitude dependence of magnetic storm ranges near sunspot maximum.

ing to the latitudes of perigee of the low-inclination satellites from which the geomagnetic storm effects for nearly all satellites except Injun III have been derived) equal to 1 . A curve was also constructed for a year of low geomagnetic activity, 1962, but it was very similar, so it is not shown.

In order to determine the latitude dependence of geomagnetic activity at lower levels of disturbance, Figure 4 was constructed from the data of Vestine et al. (1947) on the daily range at many stations during the Second International Polar Year (1932-33), which was a year of minimal geomagnetic and solar activity. The curves show the largest component of the daily range which was exceeded on $1,10,50$, and 90 percent of the days, respectively. Not having the values of $A_{p}$ for 1932-33, the writer constructed a histogram from the data on $A_{p}$ for 1954 (another year of minimal activity), smoothed it, and found that the four curves in Figure 4 correspond approximately to $A_{p}$ being greater than $43,22,9$, and 5 , respectively. Therefore, the 1 percent curve corresponds to small geomagnetic storms, several of which indeed, occurred during the Polar Year (Vestine et al., 1947). It is not surprising that the 1 percent curve closely resembles Figure 3, because they both apply to storms. The four curves in Figure 4 illustrate the well-known spread of the disturbed region to lower latitudes during storms. The latitude of maximal disturbance, however, remains at, or very close to, $69^{\circ}$.

The application of Figure 3 to the effect of geomagnetic storms on atmospheric temperature and density rests on the following observation: For a wide range of atmospheric 


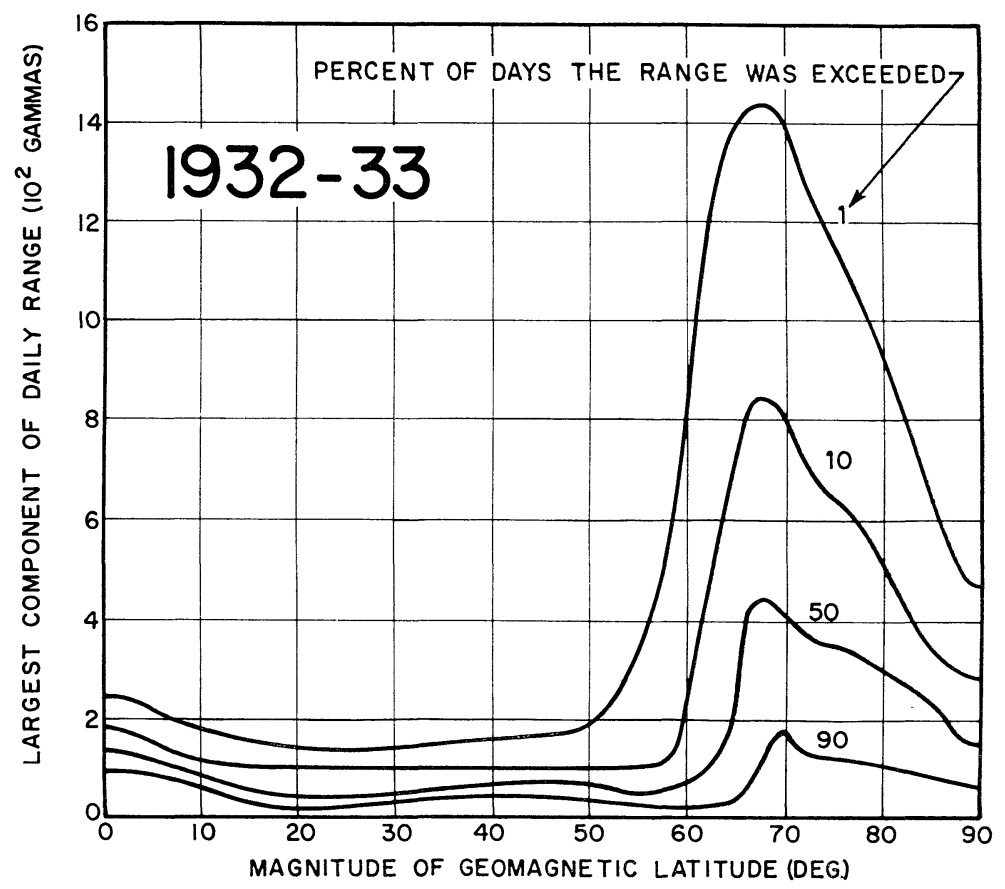

Fig. 4. Latitude dependence of daily range at sunspot minimum.

conditions, the increase in exospheric temperature during a geomagnetic storm is proportional to $A_{p}$ or $a_{p}$, according to measurements of the drag of many satellites whose perigees were at geomagnetic latitudes below $50^{\circ}$ (Jacchia, 1962; Paetzold, 1963). From this result, it appears that the temperature increase at these latitudes is proportional to the disturbance current. If the temperature increase at high latitudes is also proportional to the disturbance current, then the temperature increase at any latitude during a storm should be proportional to the curve in Figure 3. Moe (1964) has shown that if this assumption is made and the resulting temperature increases averaged over the geomagnetic latitudes at which Injun III experienced significant air drag, the computed temperature increase is consistent with the experimental result by Jacchia and Slowey (1963 b). Nevertheless, Figure 3 is not the only curve which would be consistent with this experimental result, so it seems desirable to investigate the latitude dependences of other phenomena related to storms.

\section{The Latitude Dependences of Other Atmospheric Phenomena which are Related to Geomagnetic Storms}

Figure 5 compares the latitude dependences of some related phenomena with that of the storms themselves:

(a) The average total impulse of geomagnetic micropulsations for the three largest storms during the Second Polar Year, computed from the data of Vestine et al. (1947). This curve is undependable, because the ratio of the amplitude in the auroral zone to that at low latitudes differed by a factor of ten in two of the storms.

(b) The increase in electron temperature on disturbed days, $\Delta T_{e}$, is shown at 100,150 , 
and $200 \mathrm{~km}$ (Hirao, 1963). Notice that this curve peaks at $50^{\circ}$ geomagnetic latitude, whereas the other curves peak in the auroral zone.

(c) The storm ranges from Figure 3 are reproduced.

(d) The increase in exospheric temperature, $\Delta T_{N}$, derived from the drag of satellites with perigees at high and low latitudes during storms is graphed (Jacchia 1962, Paetzold, 1963, Jacchia and Slowey, 1963 b). The horizontal lines show the approximate ranges of latitude over which the drag was experienced.

(e) The incidence of auroral forms is copied from Davis (1962 a, 1962 b). The production of light by the visible aurora requires energies of the order of ten electron volts, whereas the neutral and electron temperatures are equivalent to tenths of electron volts.

All of the disturbance phenomena shown in Figure 5 peak in the Auroral Zone except for the electron temperature increase, $\Delta T_{e}$; therefore, the electron temperature will be further considered. The electron temperatures at Wallops Island and Ft. Churchill (geomagnetic latitudes $49^{\circ}$ and $69^{\circ}$, respectively) are shown in Figure 6 (Brace and Spencer,

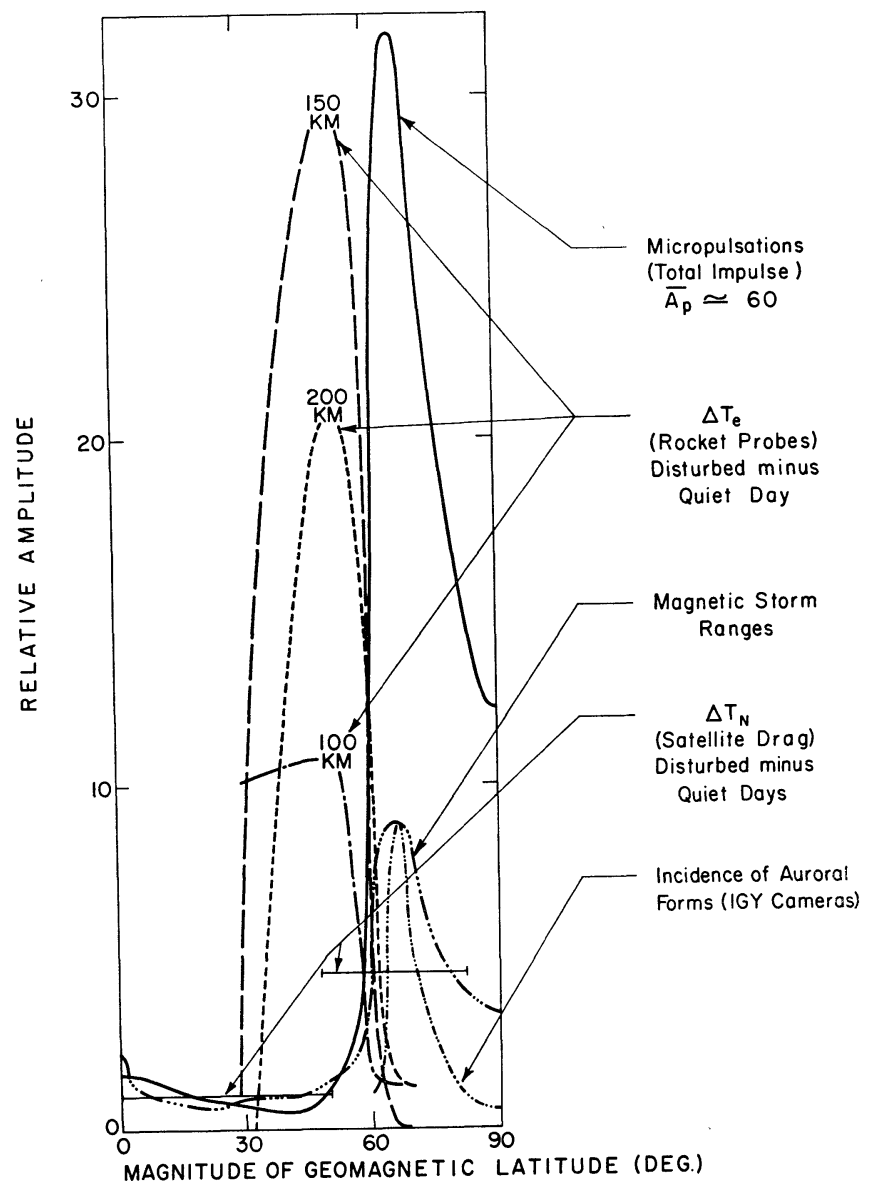

Fig. 5. Latitude dependences of phenomena related to magnetic storms. 
1964 a), for quiet and disturbed days. As Brace and Spencer show, the only curve of the four which agrees with the theories of Hanson and Johnson (1961) and Dalgarno et al. (1963) is the curve for quiet conditions at Wallops Island. The other three curves apparently require a corpuscular heat source in addition to the ultraviolet source assumed in the available theories. Another interesting feature of these curves is that on a disturbed day, the electron temperature at Ft. Churchill does not increase, but instead it becomes irregular, suggesting turbulence.

More recent evidence from the Ariel satellite (Willmore, 1964) and Explorer 17 (Brace and Spencer, $1964 \mathrm{~b}$ ) have confirmed that at geomagnetic latitudes below about 60, solar ultraviolet radiation is the only important heat source on geomagnetically quiet days (but not at night). This is consistent with the lowest curve of Figure 4. The quoted studies point out

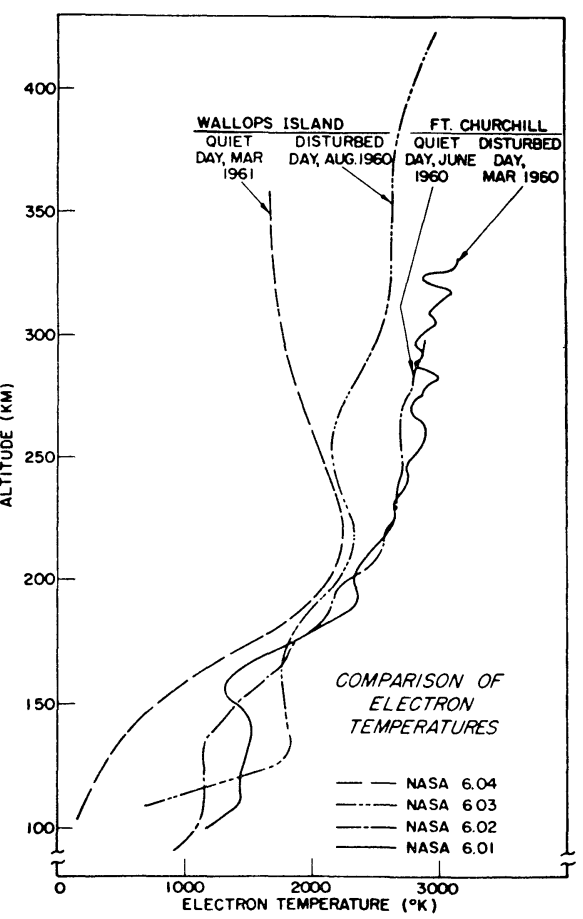

Fig. 6. Electron temperature profiles from daytime flights (after Brace and Spencer) that the increasing electron temperature with latitude at mid-latitudes on quiet days reflects a decreasing electron density, rather than an increasing energy input. New methods of studying ionograms have also yielded significant information: Becker (1964) has shown that the real height and the thickness of the $F_{2}$ layer at a mid-latitude station at noon are closely correlated with the decimeter solar flux and geomagnetic planetary amplitude, hence with the density and temperature of the neutral atmosphere deduced from satellite drag (Jacchia, 1962, Paetzold, 1963). Wright (1964) has shown that the monthly average $F_{2}$-layer thickness for quiet nights declined in phase with solar activity during 1959-1961, and that it was little affected by the monthly average $A_{p}$ outside the auroral zone, but that at Thule $\left(88^{\circ}\right.$ geomagnetic latitude) it was very strongly affected. He also showed that the monthly means of the diurnal maximum values of $F_{2}$ layer thickness were strongly dependent on the average daily insolation outside the auroral zone, but much less affected at Thule; in fact, the $F_{2}$ layer thickness at Thule was always greater than at the low-latitude stations, even when Thule was receiving one-fifth as much insolation. These results deduced from rocket and ionosonde data demonstrate the importance of corpuscular heating in the auroral atmosphere, but the data cannot be converted to neutral temperatures and densities because of the unavailability of theoretical or experimental information on the relationships among the electron, ion, and neutral temperatures in the auroral zone (Brace and Spencer, 1964 a). From the fact that the value of $\Delta T_{N}$ in Figure 5 was measured by many satellites which covered different parts of the range $0^{\circ}-50^{\circ}$ 
of geomagnetic latitude, however, and gave the same heating effect for the neutral gas, it can be concluded that $\Delta T_{e}$ in that latitude range has little effect on $\Delta T_{N}$ during storms. Although the ratio of electron-to-neutral temperature near $150 \mathrm{~km}$ during storms has not been reported, even at these latitudes, Brace and Spencer (1964 b) reported ratios as high as 3 to 1 at about $300 \mathrm{~km}$ during April 1963, which was rather quiet. It seems possible that non-equilibrium could also exist below $200 \mathrm{~km}$ during disturbed periods. From these considerations, it appears likely that the latitude dependence of the heating during storms does not follow the $\Delta T_{e}$ curve in Figure 5. Of the remaining curves, micropulsations have small amplitudes, so they are unlikely to involve much energy, while the visible aurora involves photons emitted at energies much above thermal. The latitude dependence of processes at thermal energies could be quite different. This leaves the geomagnetic storm curve as the most likely one of those in Figure 5. It has already been shown to be consistent with the Injun III data.

\section{The Energy Balance in the Auroral Zone}

If the atmospheric heating has the same latitude dependence as geomagnetic storms, large energy fluxes are required in the Auroral Zone. In response to a suggestion by G.J.F. MacDonald (private communication), a calculation has been performed (Moe, unpublished) to determine whether the energy which is observed in the Auroral Zone in various forms during a large geomagnetic storm $\left(A_{p}=200\right)$ is sufficient to produce the corresponding changes in temperature and density. The changes in temperature and density were computed from Paetzold's (1963) model atmosphere, by extrapolating that model down to $100 \mathrm{~km}$, below which the temperature and density were assumed constant. It was assumed that the total mass above $100 \mathrm{~km}$ is constant, and the changes in temperature and density are smooth functions of altitude. The increase in internal energy of the gas, the work done by the atmosphere in expanding, and the increase in potential energy were then calculated. These energies were then multiplied by a factor of 9, to convert the low-latitude conditions in Paetzold's model to conditions near the center of the Auroral Zone, giving for the increase in energy of a one-square-centimeter column $4 \times 10^{5}$ ergs when $A_{p}=200$.

Estimates of the available energy in the Auroral Zone were made from McIlwain's (1960) data on particulate fluxes, Campbell and Ree's (1961) estimate of the energy flux in a strong pulsating aurora, Maeda and Watanabe's (1963) theory of pressure waves in the Auroral Zone, O'Brien and Taylor's (1964) measurements of the average influx of particulate energy, and Chamberlain's (1961) data on Auroral brightness. These data related to different values of $A_{p}$ and different regions, so they have been extrapolated to give average conditions near the center of the Auroral Zone when $A_{p}=200$. The resulting energy flux is $300 \mathrm{ergs} / \mathrm{cm}^{2}$ sec, with an uncertainty of a factor of 5. Using Chamberlain's (1961) theoretical estimate that 15 percent of the energy is converted to heat, $45 \mathrm{ergs} / \mathrm{cm}^{2} \mathrm{sec}$ of heat are available to produce the changes in atmospheric density and temperature. From the fact that the atmospheric changes lag changes in $a_{p}$ by 5 hours (Jacchia and Slowey, 1963 a) when $a_{p}$ is declining as well as increasing, it appears that energy which is deposited in the Auroral Zone 
during storms can be dissipated in 5 hours. Therefore, enough energy must enter the Auroral atmosphere in that time to produce the observed effects. $8 \times 10^{5} \mathrm{ergs} / \mathrm{cm}^{2}$ of heat is then available, whereas $4 \times 10^{5}$ is required to produce the expected atmospheric changes.

Because of the uncertainity of a factor of 5 in the incoming corpuscular energy, the strongest statement that can be made is that the available energy is of the correct order of magnitude. These results are in agreement with Cole's (1962 a) theoretical calculation that $5 \mathrm{ergs} / \mathrm{cm}^{2} \mathrm{sec}$ of heat are generated in the Auroral Zone during moderate geomagnetic disturbances, and an order of magnitude more during large storms.

\section{References}

Becker, W., Electron Density Distribution in Ionosphere and Exosphere, E. Thrane, ed., North-Holland, Amsterdam, 199, 1964.

Brace, L.H., and N.W., Spencer, Electron Density Distribution in Ionosphere and Exosphere, E. Thrane, ed., North-Holland, Amsterdam, 88, 1964 a.

Brace, L.H., and N.W. Spencer, J. Geophys. Res., 69, 4686, 1964 b.

Campbell, W.H., and M.H. Rees, J. Geophys. Res., 66, 41, 1961.

Campbell, W.H., and J.M. Young, J. Geophys. Res., 68, 5909, 1963.

Chamberlain, J.W., Physics of the Aurora and Airglow, Academic Press, N.Y., 253, 298, and 508, 1961.

Cole, K.D., Austr. J. Phys., 15, 223, 1962 a.

Cole, K.D., Nature, 194, 761, 1962 b.

Dalgarno, A., M.B. McElroy, and R.J. Moffett, Planet. Space Sci., 11, 463, 1963.

Davis, T.N., J. Geophys. Res. 67, 59-74, 1962 a.

Davis, T.N., J. Geophys. Res., 67, 75-110, 1962 b.

Dessler, A.J., J. Geophys. Res., 64, 397, 1959.

Groves, G.V., Proc. Roy. Soc., A263, 212, 1961.

Hanson, W.B., and F.S. Johnson, Mem. Soc. R. Sci. Liege, IV, 390, 1961.

Hirao, K., J. Geomag. Geoelect., 14, 208, 1963.

Jacchia, L.G., Smithsonian Astrophys. Obs. Sp. Rept. No. 62, 1961.

Jacchia, L.G., Variations in the Earth's Upper Atmosphere as Revealed by Satellite Drag, Smithsonian Astrophys. Obs., Sp. Rept. No. 31, 1962.

Jacchia, L.G., and J. Slowey, Smithsonian Astrophys. Obs. Sp. Rept. No. 125, 1963 a.

Jacchia, L.G., and J. Slowey, Smithsonian Astrophys. Obs. Sp. Rept. No. 136, 5, 1963 b.

Jacchia, L.G. and J. Slowey, Smithsonian Astrophys. Obs. Sp. Rept. No. 152, 1964.

Maeda, K., and T. Watanabe, Infrasonic Waves from the Auroral Zones, NASA-GSFC Rept. X-640-63247, 1963.

May, B.R., Planet. Space Sci., 12, 1179, 1964.

McIlwain, C.E., J. Geophys. Res., 65, 2727, 1960.

Meadows, E.B., and J.W. Townsend, Jr., IGY Rocket Rept. 6, 87, 1960.

Moe, K., Nature, 201, 481, 1964.

Newton, G., R. Horowitz, and W. Priester, J. Geophys. Res., 69, 4690, 1964.

O’Brien, B.J. and H. Taylor, J. Geophys. Res., 69, 45, 1964.

Paetzold, H.K., in Priester, W., Space Research III, North-Holland, Amsterdam, 29, 1963.

Smelt, R., Dissertation-Stanford University, University Microfilms, Ann Arbor (Catalog No. 62-328), 1961.

Vestine, E.H., I. Lange, L. Laporte, and W.E. Scott, The Geomagnetic Field, Carnegie Institution, Publ. 580, Washington, D.C., 277, 314, and 378, 1947. 
Willmore, A.P., Nature, 202 (4927), 41, 1964.

Wright, J.W., Electron Density Distribution in Ionosphere and Exosphere, E. Thrane, ed., North-Holland, Amsterdam, 188-192. 